\title{
ESTABELECIMENTOS VETERINÁRIOS: ANÁLISE DA DISTRIBUIÇÃO POR REGIÃO DE PLANEJAMENTO E BAIRROS NO MUNICÍPIO DE NITERÓI, RJ
}

\author{
Flavio Fernando Batista Moutinho \\ Universidade Federal Fluminense, Faculdade de Veterinária e Fundação Municipal de Saúde \\ Centro de Controle de Zoonoses, Niterói, RJ, Brasil \\ flaviomoutinho@id.uff.br \\ Fábio Villas Boas Borges \\ Fundação Municipal de Saúde \\ Centro de Controle de Zoonoses, Niterói, RJ, Brasil \\ fabiovillas@gmail.com
}

\begin{abstract}
RESUMO
A Geografia da Saúde permite avaliar a organização e localização territorial dos serviços de saúde. Assim, o objetivo deste estudo foi descrever a distribuição dos estabelecimentos veterinários no município de Niterói, RJ. Para isso, foram utilizadas as seguintes categorias: estabelecimentos de atendimento direto à saúde animal (consultórios, clínicas e hospitais veterinários) e estabelecimentos de serviços especializados (serviços de diagnóstico por imagem, bancos de sangue e laboratórios de análises clínicas). Os dados foram analisados com técnicas de estatística descritiva, também confeccionados mapas com a distribuição dos estabelecimentos por região, por bairros e sobreposta à malha viária. Foram realizadas análises de correlação linear da quantidade de estabelecimentos, com a renda mediana per capita e tamanho da população canina, por bairro. A região com maior concentração de estabelecimentos veterinários, clínicas e consultórios foi Praias da Baía. O bairro com a maior concentração de estabelecimentos veterinários, clínicas e consultórios foi Icaraí. Havia dois hospitais veterinários, em São Francisco e Fonseca. Havia correlação forte da presença de estabelecimentos com a população, mas não com a renda. Nas regiões de maior urbanização e densidade populacional, os estabelecimentos veterinários encontravam-se mais dispersos pelo território, enquanto nas de urbanização moderada e características mais residenciais havia maior concentração nas vias arteriais principais.
\end{abstract}

Palavras-chave: Saúde animal. Medicina veterinária. Estabelecimentos de saúde. Espacialização. Distribuição geográfica.

\section{VETERINARY FACILITIES: ANALYSIS OF PLANNING REGION AND NEIGHBORHOOD DISTRIBUTION INT THE MUNICIPALITY OF NITERÓI - RJ}

\begin{abstract}
Health Geography allows to assess the organization and territorial location of health services. The objective of this study was to describe the distribution of veterinary es facilities in the municipality of Niterói. The following category of facilities are covered herein: facilities providing direct health care services to animals (veterinary offices, clinics and hospitals) and facilities providing specialized services (image diagnosis services, blood banks and clinical analysis laboratories). The collected data was analyzed by using descriptive statistics techniques; maps were produced to show the geographical distribution of such facilities by regions and districts, and then they were placed over the road network. Correlation analyses between the number of facilities and the average per capita income and the size of human population per district were carried out. The region with the greatest concentration of veterinary facilities, clinics and offices was Praias da Baía. The district with the greatest number of veterinary facilities, clinics and offices was Icaraí. There were two veterinary hospitals, one in São Francisco and another in Fonseca. There was a strong correlation between the existence of said facilities with the population, but not with income. Veterinary facilities were more disperse throughout the territory in more urbanized and populated regions, while they were more concentrated in the main pathways in those regions with moderate urbanization and more residential characteristics.
\end{abstract}

Keywords: Animal health. Veterinary medicine. Health care facilities. Spatialization. Geographical distribution. 


\section{INTRODUÇÃO}

A Geografia da Saúde é uma área do conhecimento que permite, dentre outras utilidades, avaliar a organização e a localização dos serviços de saúde no território. Nesse contexto, estuda-se a distribuição dos estabelecimentos de saúde no espaço e a questão do acesso da população a esses estabelecimentos. De modo geral, os serviços de saúde atraem a população mais próxima (área de influência) mas podem atrair, também, pessoas residentes em áreas mais distantes, que podem ser atraídas por fatores específicos relacionados à qualidade e à quantidade dos serviços oferecidos no estabelecimento (SANTANA, 2014).

Os animais de companhia, atualmente, são considerados por muitas pessoas como membros da família, sendo incluídos, inclusive, em ritos sociais como comemorações de aniversário. São as famílias multiespécies, em que a ética do cuidado é invocada para com a responsabilidade em relação a esses animais (CHAVES, 2017). E os humanos tendem a direcionar para seus animais a crescente demanda por serviços de saúde visando melhorar e prolongar a vida deles (CAVANAUGH et al., 2008).

Um fato muito importante que deve ser levado em consideração nessa relação estreita dos humanos com os animais diz respeito à saúde coletiva, que é o risco de transmissão das chamadas zoonoses. Trata-se das doenças transmissíveis entre os animais vertebrados e os humanos, as quais representam uma grande parcela das doenças infecciosas que vêm afetando as pessoas nos últimos anos, com destaque para as enfermidades emergentes e re-emergentes (GRISOTTI, 2010; ZANELLA, 2016; FAO/OIE/OMS, 2019).

Nesse contexto de família multiespécie, a acessibilidade em relação aos estabelecimentos de saúde animal pode ser muito importante para salvar a vida de um animal em situações de urgência e emergência ou mesmo para simples ações preventivas como vacinação e coleta de amostras biológicas para exames.

Acessibilidade diz respeito à relação existente entre a localização dos clientes, a existência de transporte, a distância e o tempo de viagem, além do custo para que se tenha acesso ao serviço de saúde (SANTANA, 1995).

O deslocamento do indivíduo para acessar um serviço é influenciado diretamente pela capacidade que a área onde está localizado tem de suprir as necessidades e a capacidade de atração está relacionada a fatores como renda, ao tamanho da população, à acessibilidade e à mobilidade urbana (MELLO e MELLO, 2013).

O ideal é que a oferta de serviços de saúde seja adequada às necessidades de cada região, assegurando acesso uniforme a esses serviços, mas geralmente as regiões mais ricas dispõem de mais e melhores serviços (SANTANA, 2014).

Os estabelecimentos veterinários são aqueles onde são realizadas intervenções médico-veterinárias, podendo ser divididos em ambulatórios, consultórios, clínicas, hospitais e aqueles de serviços veterinários especializados (CRMV-RJ, 2014; CFMV, 2019).

Os consultórios veterinários são estabelecimentos destinados à consulta clínica, onde podem ser realizados procedimentos ambulatoriais e aplicação de vacinas, não sendo permitido o uso de anestesia geral, a realização de cirurgias e internações. O mesmo ocorre com os ambulatórios, só que esses têm como característica serem dependentes de estabelecimentos industriais, comerciais, de pesquisa, de recreação, etc e atenderem exclusivamente animais pertencentes ao próprio estabelecimento. As clínicas veterinárias são destinadas às consultas e a tratamentos clínicoambulatoriais, podendo realizar cirurgias e internação. Os hospitais têm que desenvolver todas as atividades que as clínicas desenvolvem, inclusive cirurgia e internação, mas têm que oferecer também exames diagnósticos e atendimento 24 horas (CFMV, 2019).

Já os serviços veterinários especializados são os estabelecimentos envolvidos indiretamente com a assistência à saúde animal, como o diagnóstico por imagens, os laboratórios de análises clínicas, os bancos de sangue e de sêmen (CRMV-RJ, 2014).

Apesar dos estabelecimentos veterinários não serem enquadrados formalmente como estabelecimentos de saúde pelo Ministério da Saúde, já que somente são assim considerados aqueles onde são realizados ações e serviços de saúde humana (BRASIL, 2017), já há uma recomendação do Conselho Nacional de Saúde para que eles passem a ser enquadrados como tal (CNS, 2018). 
Embora existam diversos trabalhos abordando a questão da espacialização dos estabelecimentos de saúde humana, esse tipo de pesquisa não é comum na área da saúde animal, principalmente no Brasil. Sendo assim, o objetivo do presente estudo foi descrever e analisar a distribuição dos estabelecimentos veterinários no município de Niterói, RJ, com base nas regiões de planejamento e bairros.

\section{METODOLOGIA}

Niterói é um município da Região Metropolitana do Rio de Janeiro, localizado entre as coordenadas

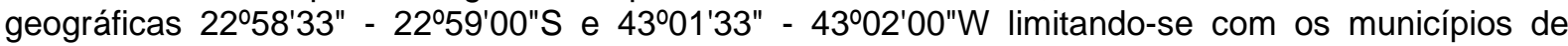
Maricá e São Gonçalo, com a baía da Guanabara e com o oceano Atlântico (IBGE, 2019). Com área de 133,916 km² (PNUD, 2013) e população de 487.562 habitantes (IBGE, 2010), Niterói apresenta Índice de Desenvolvimento Humano muito alto $(0,837)$, ocupando a $1^{\text {a }}$ colocação no Estado do Rio de Janeiro e a $7^{\text {a }}$ colocação do Brasil (PNUD, 2013).

O município é dividido, de acordo com seu Plano Diretor em cinco Regiões de Planejamento, conforme pode ser observado no Quadro 1 (NITERÓI, 2019):

Quadro 1 - Niterói (RJ): Regiões de Planejamento e respectivos bairros que as compõem.

\begin{tabular}{|l|l|}
\hline \multicolumn{1}{|c|}{$\begin{array}{c}\text { Região de } \\
\text { planejamento }\end{array}$} & \multicolumn{1}{c|}{ Bairros } \\
\hline Praias da Baía & $\begin{array}{l}\text { Boa Viagem, Cachoeira, Centro, Charitas, Fátima, Gragoatá, Icaraí, Ingá, Jurujuba, } \\
\text { Morro do Estado, Pé Pequeno, Ponta da Areia, Santa Rosa, São Domingos, São } \\
\text { Francisco, Viradouro, Vital Brazil }\end{array}$ \\
\hline Norte & $\begin{array}{l}\text { Baldeador, Barreto, Caramujo, Cubango, Engenhoca, Fonseca, Ilha da Conceição, } \\
\text { Santa Bárbara, Santana, São Lourenço, Tenente Jardim, Viçoso Jardim }\end{array}$ \\
\hline Pendotiba & $\begin{array}{l}\text { Badu, Cantagalo, Ititioca, Largo da Batalha, Maceió, Maria Paula, Matapaca, Sapê, } \\
\text { Vila Progresso }\end{array}$ \\
\hline Leste & Muriqui, Rio do Ouro, Várzea das Moças \\
\hline Oceânica & $\begin{array}{l}\text { Cafubá, Camboinhas, Engenho do Mato, Itacoatiara, Itaipu, Jacaré, Jardim Imbuí, } \\
\text { Maravista, Piratininga, Santo Antônio, Serra Grande }\end{array}$ \\
\hline
\end{tabular}

Para a realização da pesquisa foi utilizado o banco de dados de estabelecimentos veterinários mantido pelo Centro de Controle de Zoonoses de Niterói.

Os dados dos diferentes estabelecimentos veterinários, foram classificados por categoria, região, bairro e coordenadas geográficas de localização (coletadas por geocodificação de endereços), sendo então armazenados em uma planilha $\operatorname{Excel}^{\circledR}$

Duas categorias de estabelecimentos veterinários foram utilizadas:

Estabelecimentos de atendimento direto à saúde animal: incluem consultórios, clínicas e hospitais veterinários.

Estabelecimentos de serviços especializados: incluem os serviços de diagnóstico por imagem (raios $\mathrm{x}$ e/ou tomografia computadorizada), bancos de sangue e laboratórios de análises clínicas veterinárias.

Importante destacar que não havia nenhum ambulatório no banco de dados e que alguns estabelecimentos de atendimento direto à saúde animal prestam serviços especializados (de apoio em diagnóstico por imagem e laboratório de análises clínicas) a outros estabelecimentos de atendimento direto à saúde animal.

Os dados foram analisados com técnicas de estatística descritiva em números absolutos e proporcionais com uso das ferramentas disponíveis no programa Excel.

\begin{tabular}{|c|c|}
\hline & Uberlândia-MG \\
\hline
\end{tabular}


Também foram confeccionados três mapas temáticos utilizando-se o Sistema de Informação Geográfica (SIG) Q-Gis 3.12 Bacuresti: o primeiro mapa exibe a espacialização dos estabelecimentos por região de planejamento, o segundo exibe a espacialização por bairros e no terceiro os estabelecimentos foram sobrepostos à malha viária do município.

Por último foram realizadas as análises de correlação linear da quantidade total de estabelecimentos, clínicas e consultórios, com a renda mediana per capita e com o tamanho da população de cães por bairro, estimada conforme indicado pelo Ministério da Saúde (BRASIL, 2002), a partir da população humana do Censo do Instituto Brasileiro de Geografia e Estatísticas de 2010 (IBGE, 2010).

\section{RESULTADOS E DISCUSSÃO}

Quando este levantamento foi realizado, em janeiro de 2020, havia em Niterói: 2 hospitais, 35 clínicas e 43 consultórios veterinários, totalizando 80 estabelecimentos de atendimento direto à saúde veterinária. Considerando somente os serviços especializados, havia 1 banco de sangue, 2 estabelecimentos que prestavam exclusivamente serviço de diagnóstico por imagem e 2 estabelecimentos que eram exclusivamente laboratórios de análises clínicas. Além disso, havia 4 estabelecimentos que ofereciam serviço de atenção direta à saúde dos animais, além de serviço de diagnóstico por imagem para clientes externos. Um desses estabelecimentos oferecia, ainda, serviço para terceiros de laboratório de análises clínicas.

\section{Distribuição por regiões de planejamento}

Ao se avaliar a distribuição por regiões de planejamento, constatou-se grande concentração dos estabelecimentos de atendimentos direto à saúde animal nas regiões Praias da Baía $(n=40)$ e Oceânica $(n=24)$, quantidade intermediária nas regiões Norte $(n=8)$ e Pendotiba $(n=7)$ enquanto a região Leste contava com somente 1 estabelecimento (Figura 1).

Figura 1 - Percentual de estabelecimentos veterinários de atendimento direto à saúde animal por região de planejamento.

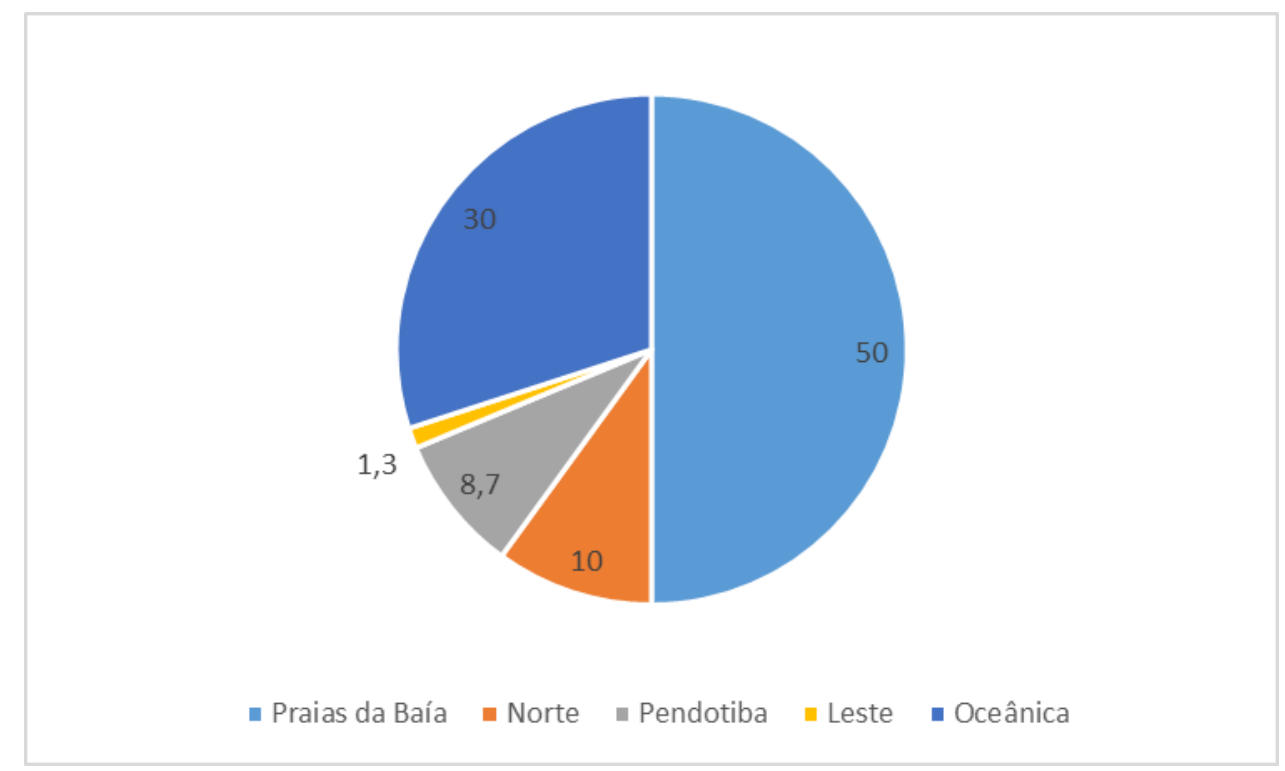

Fonte - banco de dados de estabelecimentos veterinários do Centro de Controle de Zoonoses de Niterói. Organizado pelos autores.

A figura 2 exibe distribuição concentrada de estabelecimentos nas regiões de planejamento Praias da Baía e Oceânica, em detrimento das demais regiões, principalmente da Leste. Nota-se, ainda, que mesmo os estabelecimentos exclusivos de apoio diagnóstico e complementares também se concentram nessas regiões, exceto pela existência de um banco de sangue na região de Pendotiba. 
Figura 2 - Distribuição dos estabelecimentos veterinários no município de Niterói por região de planejamento.

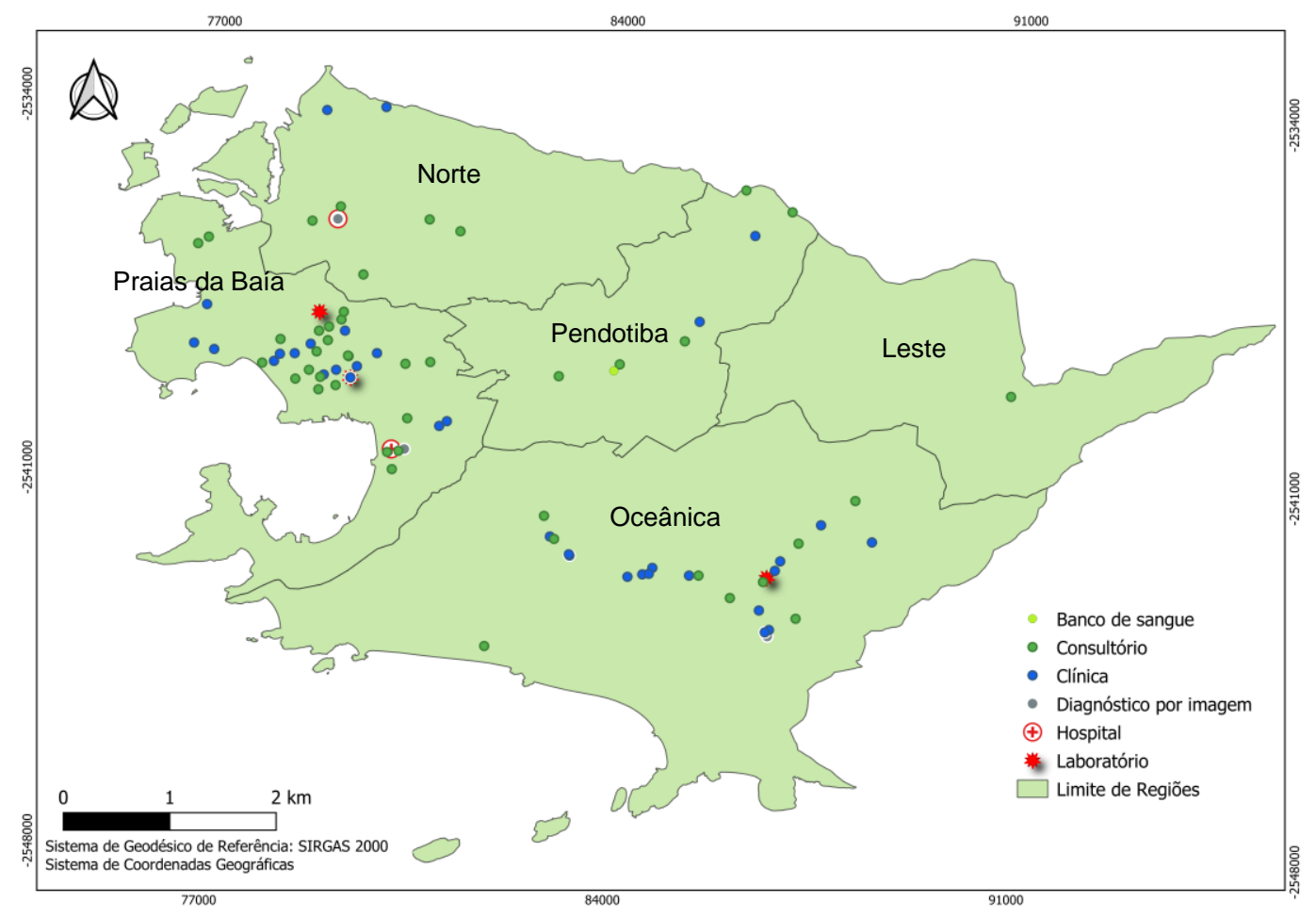

Fonte - Banco de dados de estabelecimentos veterinários do Centro de Controle de Zoonoses de Niterói. Organizado pelos autores.

Considerando somente os consultórios veterinários, estes predominam nas regiões Praias da Baía $(n=23)$ e Oceânica $(n=9)$, eram em quantidade intermediária nas regiões Norte $(n=5)$ e Pendotiba $(n=5)$ e apenas um consultório $(2,3 \%)$ estava presente na região Leste (Figura 3$)$.

Figura 3 - Percentual de consultórios veterinários por região de planejamento.

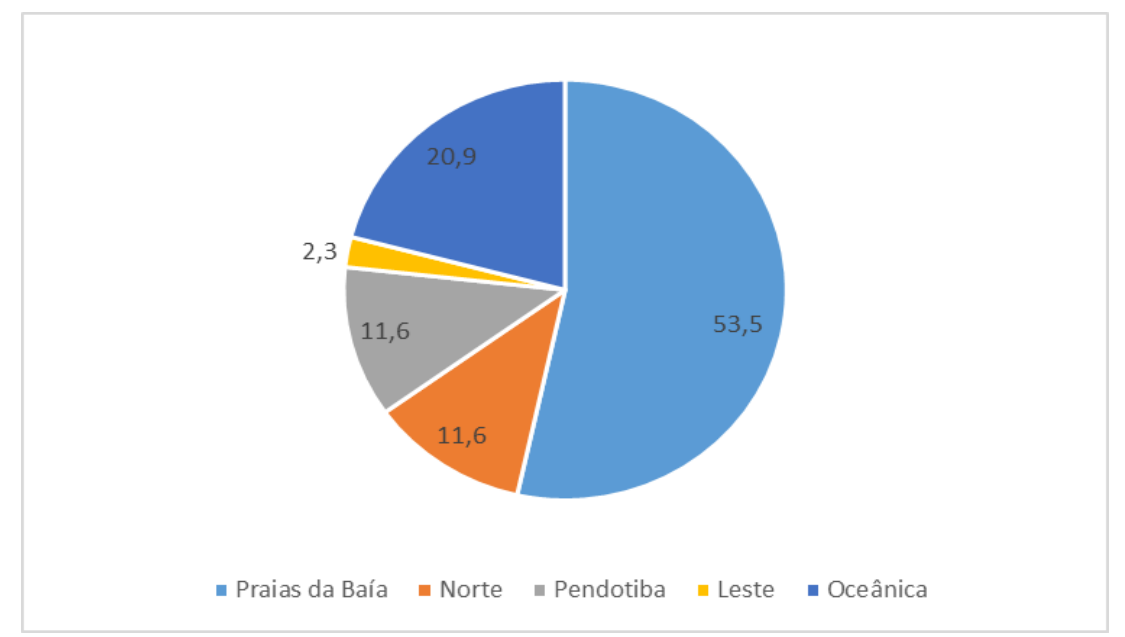

Fonte - Banco de dados de estabelecimentos veterinários do Centro de Controle de Zoonoses de Niterói. Organizado pelos autores.

Quanto às clínicas veterinárias, é verificado uma distribuição mais equilibrada entre as regiões predominantes, a Praias da Baía $(n=16)$ e Oceânica $(n=15)$ e uma quantidade substancialmente menor nas regiões Norte $(n=2)$ e Pendotiba $(n=2)$. Além disso, notou-se a inexistência de clínicas na região Leste (Figura 4). 
Figura 4 - Percentual de clínicas veterinárias por região de planejamento.

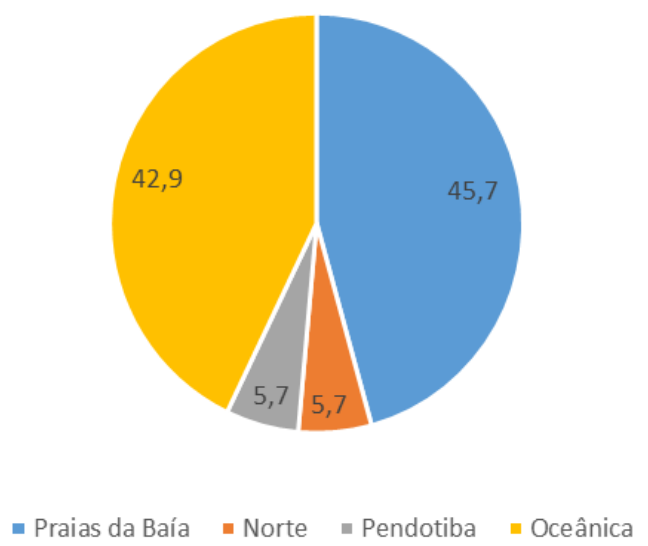

Fonte - Banco de dados de estabelecimentos veterinários do Centro de Controle de Zoonoses de Niterói.

Organizado pelos autores.

Dois hospitais veterinários estão presentes em Niterói, um localiza-se na região Praias da Baía e outro na região Norte.

Em relação à oferta de serviços especializados, novamente a concentração se repetiu nas regiões Praias da Baía e Oceânica, com 4 estabelecimentos cada (Figura 5). Na região Praias da Baía havia 2 estabelecimentos com serviço de laboratório de análises clínicas e 2 com diagnóstico por imagem; na Oceânica, um de laboratório e 3 de diagnóstico por imagem; na Norte um de diagnóstico por imagem; e na de Pendotiba um banco de sangue. Nenhum estabelecimento oferecia serviço veterinário especializado na região Leste.

Figura 5 - Percentual de estabelecimentos que ofereciam serviços veterinários especializados por região de planejamento.

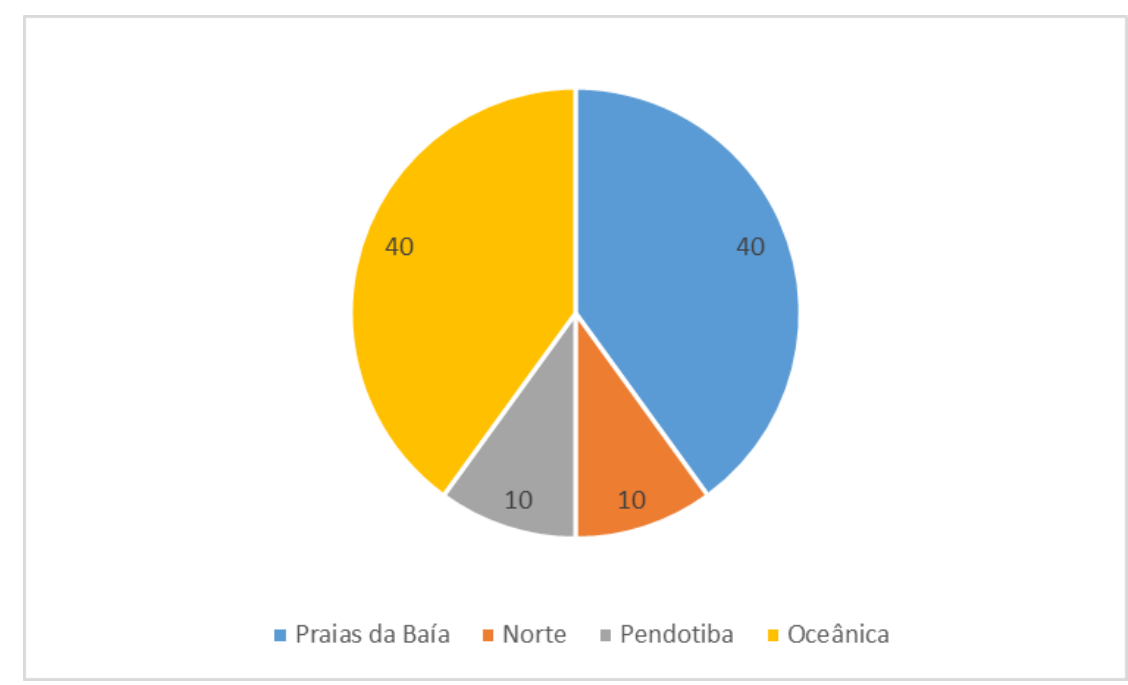

Fonte - Banco de dados de estabelecimentos veterinários do Centro de Controle de Zoonoses de Niterói. Organizado pelos autores.

Nas organizações manufatureiras o foco da sua localização geralmente está no custo de produção, mas nas empresas do setor de serviços este se desloca para fatores relacionados à clientela, que deve, via de regra, ter suas expectativas satisfeitas e, preferencialmente, superadas, tornando o processo de tomada de decisão sobre a localização muitas vezes mais complexo (DAVIS; AQUILANO; CHASE, 2001). Esse processo de tomada de decisão se mostra, muitas vezes, mais

$\begin{array}{lllll}\text { Caminhos de Geografia } & \text { Uberlândia-MG } & \text { v. 22, n. } 79 & \text { Fev/2021 } & \text { p. 321-335 Página } 326\end{array}$


subjetivo, mas nem por isso pode prescindir da racionalização e da análise das variáveis envolvidas (LOPES e ALMEIDA, 2008).

Os estabelecimentos veterinários são empresas do setor de serviços e, diferentemente das empresas agrícolas ou industriais, as empresas de serviços dependem de uma localização mais central que garanta uma procura mínima que torne o negócio economicamente sustentável; isso envolve posição geográfica, acessibilidade e um mercado mínimo que viabilize os investimentos necessários (RIBEIRO e SANTOS, 2005).

O poder de atratividade de um negócio estaria relacionado ao tipo de deslocamento que o consumidor assume em diferentes regiões, situações e momentos, bem como a variáveis como tamanho da população, renda, mobilidade urbana e acessibilidade (MELLO e MELLO, 2013).

Fazendo uma analogia do ensaio metodológico realizado por Santana (2014) mas aplicando-o à saúde animal, ao se avaliar a questão da acessibilidade aos estabelecimentos veterinários, três dimensões devem ser consideradas: território, pacientes e organização dos serviços. Em relação ao território, deve-se considerar o custo e o tempo que se leva entre a residência e o local do atendimento, ou seja, fatores relacionados ao acesso locacional. No que tange aos animais deve-se considerar fatores como idade do animal, renda do tutor, conhecimentos sobre cuidados de saúde, dentre outros. Por fim, na organização dos serviços, considera-se a especialização, as características do serviço incluindo tipo, qualidade, referências, possibilidade de continuidade nos cuidados e hierarquia dos cuidados.

A distância percorrida pelas pessoas em busca de atendimento de saúde para os animais, varia de acordo com a complexidade do atendimento oferecido. Os estabelecimentos que oferecem cuidados mais básicos ou primários, como os consultórios veterinários devem estar localizados mais próximos das residências, enquanto aqueles que oferecem cuidados mais especializados, como as clínicas veterinárias, podem impor a concentração dos serviços, implicando em deslocamentos mais longos (SANTANA, 2014). Isso ocorre porque esses serviços mais básicos envolvem, de modo geral, custos menores sendo de utilização mais frequente. Já aqueles mais especializados, por envolverem mais tecnologia, apresentam custos maiores e, geralmente, têm menor demanda, estando sujeitos a economia de escala (SIMÕES et al., 2004).

Além de atrair pacientes veterinários de sua área direta de influência, pela proximidade, os estabelecimentos de saúde podem atrair pacientes de áreas mais distantes em funções de condições específicas que eles venham a apresentar. De acordo com Santana (2014), essas condições dizem respeito a atendimento de urgência e emergência, atendimento permanente, facilidade de acesso, qualidade de instalações, qualidade do atendimento, quantidade e qualidade dos profissionais, presença de alta tecnologia, acreditação do estabelecimento, custos, satisfação com o estabelecimento, dentre outros.

Esses fatores explicam a grande concentração de estabelecimentos veterinários na região Praias da Baía. O processo de ocupação do município de Niterói teve início a partir do sistema hidroviário que ainda hoje une a cidade ao centro do Rio de Janeiro. Esse sistema era alimentado por bondes que faziam sua ligação com os demais bairros das regiões Praias da Baía e Norte, as primeiras a serem ocupadas. A região Praias de Baía passou por um processo de substituição do casario antigo de poucos pavimentos por prédios mais altos e modernos, com o bairro de Icaraí se consolidando nos anos 1970 como centro urbano catalisador e concentrador do lazer, do comércio e dos serviços (FIGUEIREDO, 2015). Esta região caracteriza-se por apresentar predomínio de áreas com urbanização intensa e muito verticalizada (LOVISI e SEABRA, 2016).

A região Oceânica, que ocupa o segundo lugar em quantidade de estabelecimentos, vem passando por intenso processo de urbanização desde os anos 1970, com crescimento da área urbana de $111,7 \%$ entre 1976 e 2011 , e crescimento de $600 \%$ no número de domicílios, alcançando quase 22.000 unidades (FONTENELLE e CORRÊA, 2012).

Esta região é responsável pela maior taxa de crescimento populacional entre todas as demais regiões do município e em 2010 já abrigava $14,2 \%$ dos munícipes (68.987 pessoas), em $52,1 \mathrm{~km}^{2}$ (38,9\% do território) (FONTENELLE e CORRÊA, 2013).

A região Norte, terceira colocada em quantidade de estabelecimentos veterinários, está historicamente associada, juntamente com a Praias da Baía, ao início do processo de ocupação de Niterói, tendo como característica a existência de conurbação com o município de São Gonçalo (FONTENELLE e CORRÊA, 2013). A região caracteriza-se por urbanização intensa com predomínio 
de famílias com menor renda e presença de favelas (LOVISI e SEABRA, 2016), o que poderia explicar um número pouco expressivo de estabelecimentos, principalmente aqueles de maior complexidade, como clínicas, apesar da existência de um hospital veterinário no bairro Fonseca.

A região de Pendotiba, penúltima região em quantidade de estabelecimentos veterinários, tem predominância residencial horizontal, com densidade demográfica bem inferior à região Praias da Baía, pouca acessibilidade a serviços e à transporte coletivo, o que torna frequente o uso de veículos para deslocamento da população (GRIECO e PORTUGAL, 2010). Nesta região predominam áreas de urbanização rarefeita a moderada com muitas áreas verdes (LOVISI e SEABRA, 2016).

A região Leste, que só dispunha de um estabelecimento veterinário, possui todos os seus bairros com urbanização rarefeita, sendo predominantemente residencial e com presença significante de áreas verdes e baixa densidade demográfica, o que poderia explicar a pequena quantidade de estabelecimentos veterinários (LOVISI e SEABRA, 2016). Não se pode descartar, também, a possibilidade que moradores das regiões Leste e Norte utilizem serviços veterinários localizados no município limítrofe de São Gonçalo, devido à proximidade de ambos.

As diferenças regionais intra-municipais foram bem estudadas por Milton Santos em 1978, o qual defendia que o espaço é um conjunto de fixos e fluxos. Esses fixos estão localizados em determinados lugares e os fluxos modificam tais lugares, redefinindo-os. Atualmente, vive-se uma época de extrema especialização em que novas técnicas mais avançadas surgem a todo momento, suplantando as anteriores por suas superioridades operacionais. Mas nas cidades, devido à durabilidade e ao custo de mudança dos fixos, há uma resistência maior a essas mudanças. Assim, enquanto em algumas áreas se instalam novas infraestruturas, vias rápidas, prédios inteligentes, em outras persistem rugosidades do passado (SANTOS, 2006).

\section{Distribuição por bairros}

Niterói está dividido em 52 bairros (NITERÓI, 2019). Em metade deles não havia nenhum estabelecimento veterinário de atendimento direto à saúde animal.

O bairro com maior quantidade de estabelecimentos veterinários de atendimento direto à saúde animal era Icaraí, com 17 estabelecimentos, seguido de Santa Rosa, com 7, ambos na região Praias da Baía (Figura 6). Em Icaraí estavam localizados 21,2\% dos estabelecimentos desse tipo de todo o município.

Figura 6 - Quantidade de estabelecimentos veterinários de atendimento direto à saúde por bairro, 2020.

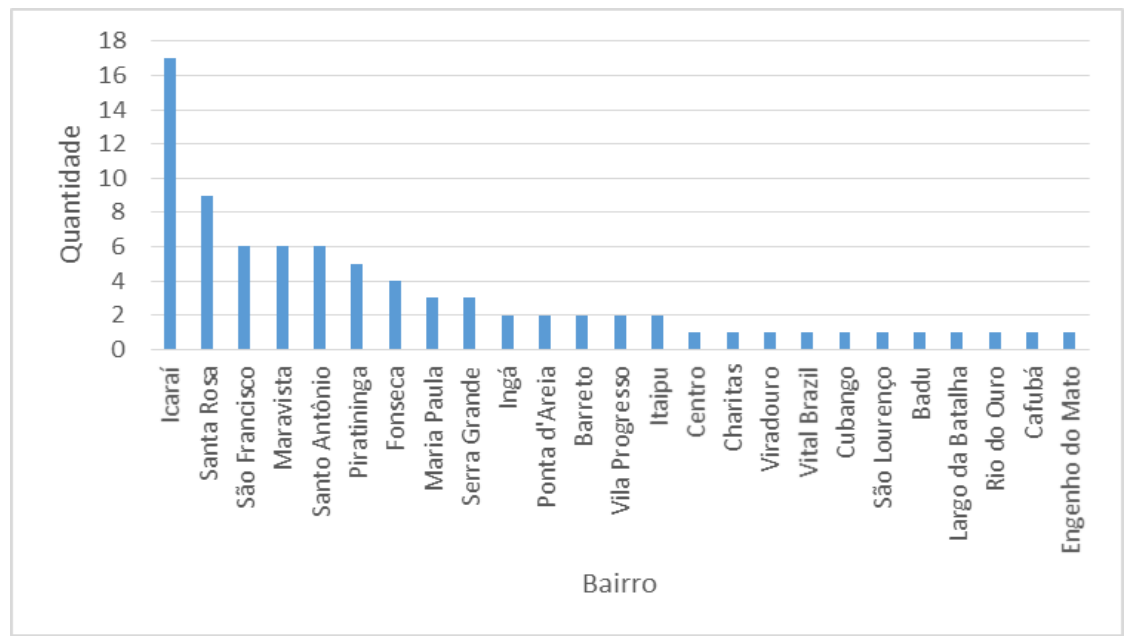

Fonte - Banco de dados de estabelecimentos veterinários do Centro de Controle de Zoonoses de Niterói Organizado pelos autores.

Quando avaliados isoladamente, os consultórios eram mais presentes, também, nos bairros Icaraí $(n=9)$ e Santa Rosa ( $n=7)$ (Figura 7). Somados, esses dois bairros limítrofes, dispunham de $40 \%$ dos 
consultórios veterinários do município e somente Icaraí contava com $22,5 \%$ deles.

Figura 7 - Quantidade de consultórios veterinários por bairro.

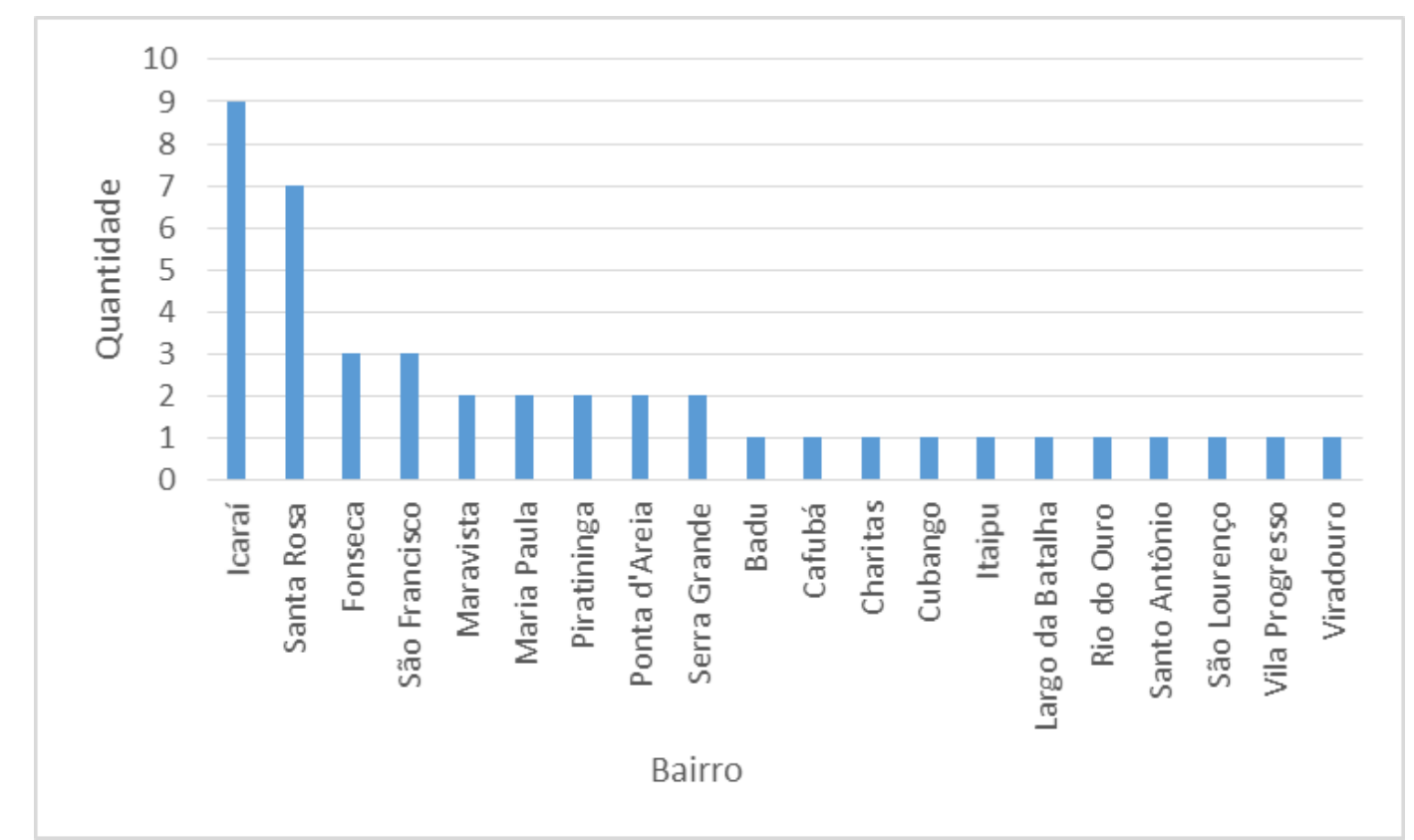

Fonte - Banco de dados de estabelecimentos veterinários do Centro de Controle de Zoonoses de Niterói. Organizado pelos autores.

Quanto às clínicas, estavam mais presentes também em Icaraí $(n=8)$, seguido do bairro Santo Antônio $(n=5)$ e Maravista $(n=4)$, ambos na região Oceânica (Figura 8). Somente em Icaraí estavam localizadas $22,8 \%$ das clínicas veterinárias de Niterói.

Figura 8 - Quantidade de clínicas veterinárias por bairro.

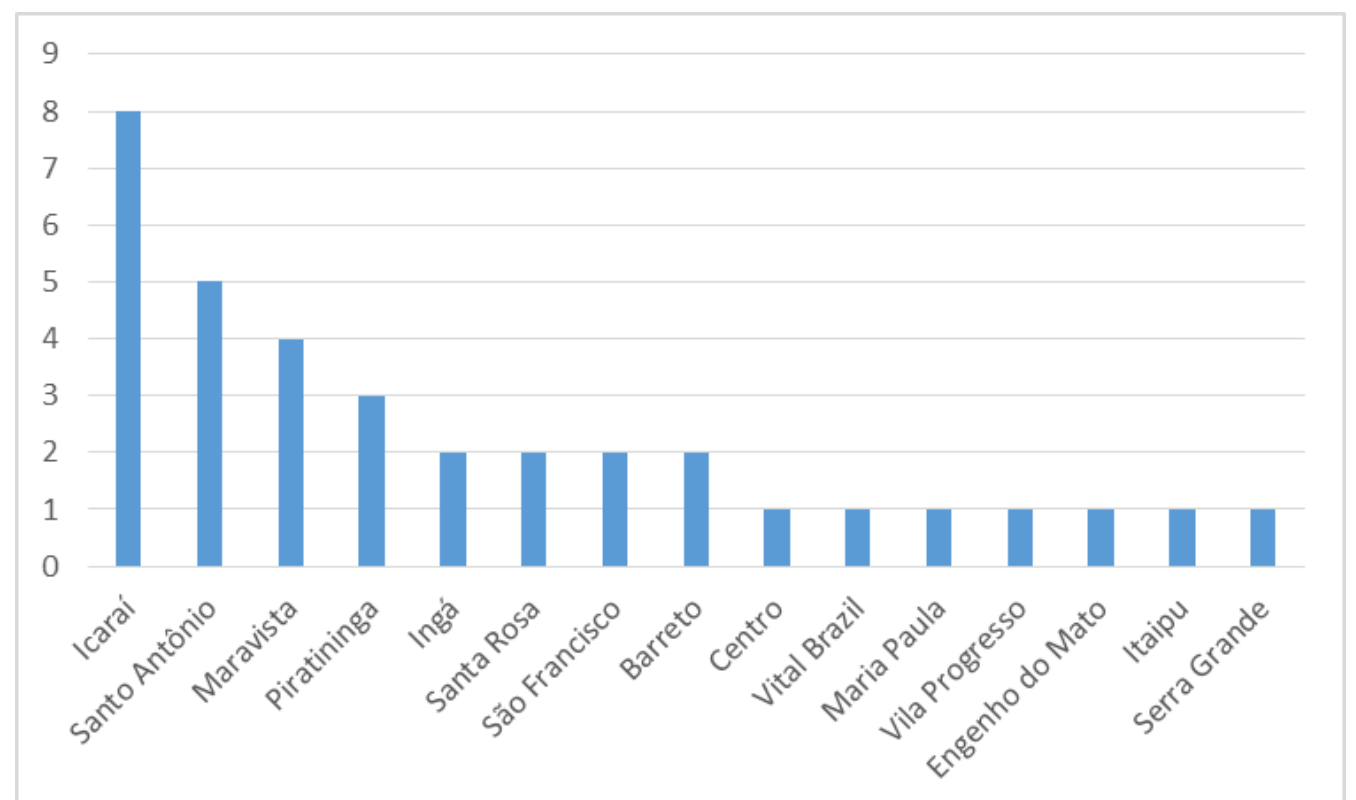

Fonte - Banco de dados de estabelecimentos veterinários do Centro de Controle de Zoonoses de Niterói. Organizado pelos autores. 
Os dois hospitais veterinários existentes no município estavam localizados nos bairros Fonseca e São Francisco.

No que diz respeito à oferta de serviços especializados, os três serviços de laboratório de análises clínicas estavam localizados em Icaraí, Pé Pequeno e Maravista. O único banco de sangue estava localizado no Badu e os serviços de diagnóstico por imagem nos bairros São Francisco, Fonseca, Icaraí, Piratininga e Itaipu $(n=2)$. Itaipu detinha $33,3 \%$ dos serviços de diagnóstico por imagem veterinária do município.

A figura 9 exibe a grande concentração de estabelecimentos veterinários no bairro Icaraí, principalmente, bem como aglomerados secundários em Santa Rosa, São Francisco, Santo Antônio e Maravista.

Figura 9 - Distribuição, por bairro, dos estabelecimentos veterinários.

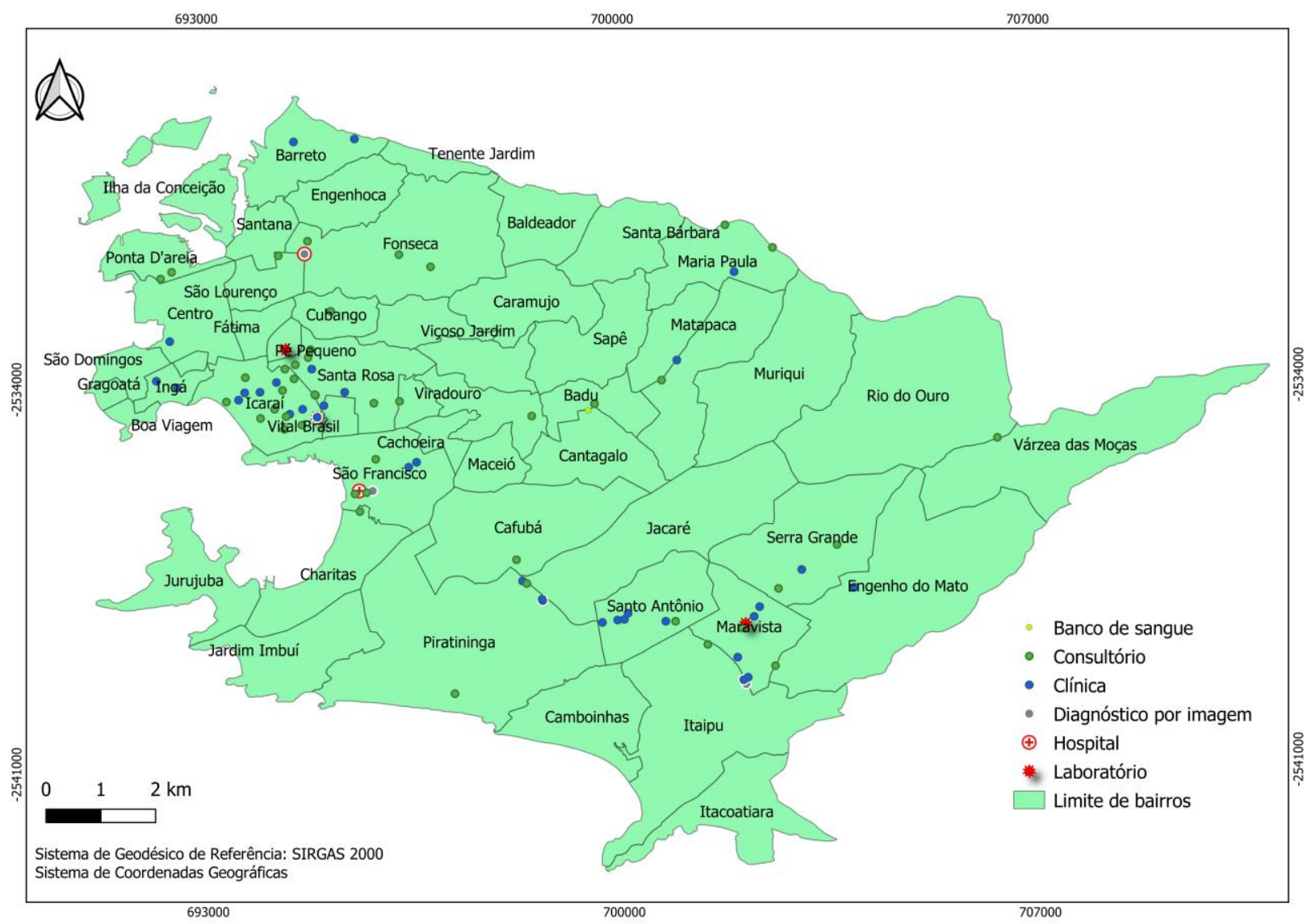

Fonte - Banco de dados de estabelecimentos veterinários do Centro de Controle de Zoonoses de Niterói. Organizado pelos autores.

De modo geral, em Niterói, as atividades econômicas se concentram no Centro e em Icaraí, bairros que apresentam localizações estratégicas e facilidade de acesso a transportes (FGV,2015). Oliveira e Mizubuti (2007) destacam que em Icaraí circula grande parte da renda do município já que o bairro se transformou em um centro de comércio e serviços. Icaraí apresenta grande densidade populacional, comércio local diversificado e muita acessibilidade aos serviços, propiciando que os moradores realizem muitos deslocamentos a pé (GRIECO e PORTUGAL, 2010). Lefebvre (2001) destaca o duplo caráter da centralidade capitalista, de lugar de consumo e de consumo de lugar. Além da densificação da possibilidade de consumo, esses centros são lugares de encontro, reunião, onde as 
pessoas vão para se ver, se olhar, se falar.

No período 2001 a 2014, dos 193 lançamentos imobiliários de Niterói, 157 ocorreram na região Praias da Baía, agregando 15.443 novas unidades domiciliares de um total de 19.611 ofertadas no período, - que reforça a mesma como região central do município, concentrando $48,7 \%$ da população niteroiense. Dos 77 empreendimentos lançados na região entre 2011 e 2014, um total de 33 se localizava em Icaraí, reforçando sua centralidade (FIGUEIREDO, 2015).

A região Oceânica vem apresentando crescimento consistente há algumas décadas e, em 2010, os bairros Maravista e Santo Antônio, que apresentaram grande quantidade de clínicas veterinárias, já apresentavam área urbanizada de $94,2 \%$ e $66,8 \%$ respectivamente (FONTENELLE e CORRÊA, 2013), reforçando que já havia uma clientela apta para fazer uso dos serviços veterinários ali estabelecidos.

$\mathrm{Na}$ segunda etapa de desenvolvimento deste trabalho foi analisada se havia correlação entre a população estimada de cães e a quantidade de estabelecimentos veterinários em cada bairro, tendo sido encontrada correlação linear de $0,78(p<0,0001)$, que pode ser considerada forte de acordo com Barbetta (2012). A correlação linear entre a população estimada de cães em cada bairro e a quantidade de consultórios também foi de $0,78(p<0,0001)$. Já a correlação linear entre a população estimada de cães em cada bairro e a quantidade de clínicas foi de 0,62 ( $p<0,0001)$ e, sendo considerada moderada, de acordo com Barbetta (2012).

Também foi analisado, se havia correlação entre a renda mediana per capita e a quantidade de estabelecimentos localizadas por bairro, tendo sido encontrada correlação de $0,33(p=0,179)$, que pode ser considerada fraca de acordo com Barbetta (2012). A correlação linear entre a renda mediana per capita por bairro e a quantidade de consultórios foi ainda mais fraca, de 0,22 ( $p=0,1223$ ). Já a correlação entre a renda mediana per capita por bairro e a quantidade de clínicas veterinárias foi um pouco mais alta, 0,41 ( $p=0,0024)$, sendo considerada moderada, de acordo com Barbetta (2012). Apesar disso, nenhuma das três análises de correlação linear com a renda mediana per capita foram consideradas estatisticamente significantes.

De modo geral, os gastos com a saúde de animais de estimação aumentam conforme a renda domiciliar de seus tutores (CARVALHO e PESSANHA, 2013).E, apesar de Perobelli et al (2016) afirmarem que dentre os fatores que podem mudar radicalmente a hierarquia dos lugares centrais estão as mudanças no tamanho da população e o poder de compra dos indivíduos, os resultados não demonstraram correlação estatisticamente significante com a renda, evidenciando, todavia, forte correlação com o tamanho da população. Os mesmos autores destacam que a expansão dos serviços que são mais diretamente ligados à presença dos indivíduos em determinado território, como os de saúde, está correlacionada ao poder de gastos e ao tamanho da população e que, com o aumento de ambos, a demanda por serviços se modifica em quantidade e em variedade. Isso pode ser visualizado com os resultados obtidos para o bairro Icaraí, que tem a maior população do município, uma alta renda per capita e concentra a maior parte dos estabelecimentos veterinários, das clínicas e consultórios veterinários.

\section{Distribuição dos estabelecimentos e malha viária}

A espacialização dos estabelecimentos veterinários com a malha viária do município, permitiu observar que na região Praias da Baía, onde se concentra a maior proporção dos estabelecimentos, e na Norte, estes estabelecimentos encontram-se espalhados por diferentes vias. Mas para as demais regiões, exceto a Leste onde só há um estabelecimento veterinário, notam-se três vias arteriais principais que se destacam, onde há uma visível concentração de estabelecimentos. $\mathrm{Na}$ região Pendotiba, sobressaem a Estrada Caetano Monteiro e na região Oceânica, as avenidas Francisco da Cruz Nunes e Ewerton Xavier (Figura 10).

\begin{tabular}{|c|c|c|c|}
\hline afia & \multicolumn{2}{|l|}{ Uberla } & Fev/2021 \\
\hline
\end{tabular}


Figura 10 - Malha viária e distribuição dos estabelecimentos veterinários.

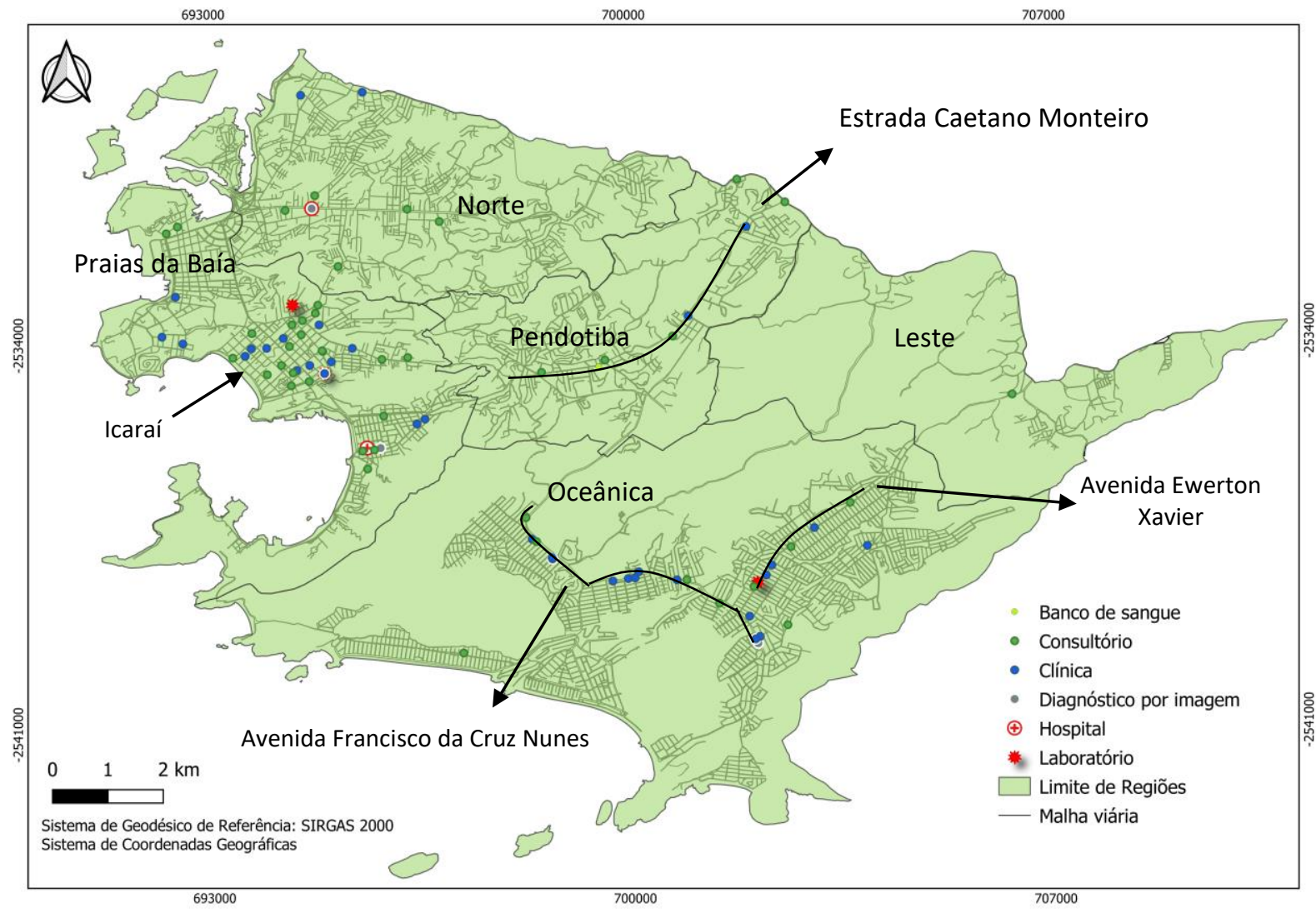

Fonte - Banco de dados de estabelecimentos veterinários do Centro de Controle de Zoonoses de Niterói. Malha viária: site do IBGE disponível em http://geoftp.ibge.gov.br/. Organizado pelos autores.

Esses resultados podem ser explicados pela grande densidade demográfica e a característica da centralidade de Icaraí, bem como pela acessibilidade dos estabelecimentos, inclusive para deslocamentos a pé (OLIVEIRA e MIZUBUTI, 2007; GRIECO e PORTUGAL, 2010). Outro fator a ser considerado é a grande urbanização das regiões Norte e Praias da Baía (LOVISI e SEABRA, 2016). No entanto, as regiões Pendotiba e Oceânica apresentam características de urbanização rarefeita a moderada, com predomínio residencial o que direcionam as atividades comerciais e de prestação de serviços a se fixarem nas vias arteriais principais, mais acessíveis (LOVISI e SEABRA, 2016).

\section{CONSIDERAÇÕES FINAIS}

O presente estudo permitiu tecer algumas considerações interessantes sobre a regionalização dos estabelecimentos veterinários em Niterói.

Inicialmente é importante destacar a centralidade que a região Praias da Baía e, principalmente, o bairro Icaraí e seus limítrofes representam para esse tipo de estabelecimento no município. Nela estão localizados a maioria dos consultórios e clínicas veterinários do município, bem como ela divide a primeira colocação com a região Oceânica em quantidade de estabelecimentos de serviços especializados, o que pode ser evidenciado pela espacialização dos dados (Figura 9)

Segue-se em importância a região Oceânica, em quantidade de consultórios e clínicas veterinárias, a Norte e Pendotiba apresentam-se como intermediárias, e a Leste apresenta uma grande lacuna de estabelecimentos com somente um consultório veterinário. Esta, por conseguinte, está localizada em uma área rica em fragmento de mata e tem escassa malha viária, o que justificaria a ausência de estabelecimentos.

Deve-se destacar, também, a importância do tamanho da população estimada de cães na possível 
localização dos estabelecimentos veterinários, tendo havido forte correlação linear. A correlação linear entre a quantidade de estabelecimentos veterinários e a renda se mostrou fraca, mas não foi significante estatisticamente. Acredita-se que na ausência de estabelecimentos veterinários em seus bairros de residência, as pessoas residentes em bairros com menor renda se desloquem para atendimento de seus animais nos bairros onde eles estejam localizados. Além disso, os bairros com renda mais alta, como Itacoatiara e Camboinhas, são bairros com características estritamente residenciais e com população residente pequena.

Cabe salientar que, nas regiões e bairros onde a urbanização é mais intensa os estabelecimentos veterinários tendem a ter uma disposição mais dispersa pelas diferentes vias (Praias da Baía e Norte), enquanto nas regiões e bairros com características mais residenciais e de urbanização mediana ou rarefeita há uma predileção por vias arteriais principais, de maior movimento (Pendotiba e Oceânica).

Por fim, é importante destacar a importância do presente trabalho em função de sua inovação ao abordar os estabelecimentos veterinários e, também, para o processo decisório de empresários em relação à localização de futuros estabelecimentos do ramo. Como ponto negativo deve ser registrado o não aprofundamento do estudo em relação aos serviços oferecidos em cada estabelecimento, que podem diferir muito de um para o outro, mesmo dentro da mesma categoria de estabelecimento.

\section{REFERÊNCIAS}

BARBETTA, P. A. Estatística aplicada às Ciências Sociais. 8 ed. Florianópolis: UFSC, 2012.

BRASIL. Ministério da Saúde, Fundação Nacional de Saúde. Encontro Nacional dos Coordenadores de Zoonoses. Relatório Anual. Brasília (DF): Ministério da Saúde; 2002.

BRASIL. Portaria Ministério da Saúde no 2.022, de 7 de agosto de 2017. Disponível em: http://www.in.gov.br/materia/-/asset publisher/Kujrw0TZC2Mb/content/id/19236814/do1-2017-08-15portaria-n-2-022-de-7-de-agosto-de-2017--19236724. Acesso em: 20 de abril de 2020.

CARVALHO, R. L. S.; PESSANHA, L. D. R. Relação entre famílias, animais de estimação, Afetividade e consumo: estudo realizado em bairros do Rio de Janeiro. Sociais e humanas, v. 26, n. 03, p. 622 637, 2013.

CAVANAUGH, L. A.; LEONARD, H. A.; SCAMMON, D. L.. A tail of two personalities: How canine companions shape relationships and well-being. Journal of Business Research, v. 61, n.5, p. 469479, 2008. https://doi.org/10.1016/i.jbusres.2007.07.024

CHAVES M. Disputa de guarda de animais de companhia em sede de divórcio e dissolução de união estável: reconhecimento da família multiespécie? In: PEREIRA, R. C.; GRISARD FILHO, W.; BURGUER, M. L. F. (Org.). Direito das famílias e sucessões: concurso de artigos. Belo Horizonte: IBDFAM, 2017, p. 51-81.

CNS - CONSELHO NACIONAL DE SAÚDE. Recomendação no 061, de 13 de dezembro de 2018. Disponível em: https://conselho.saude.gov.br/recomendacoes/2018/Reco061.pdf. Acesso em: 21 de abril de 2020.

CRMV-RJ - Conselho Regional de Medicina Veterinária do Estado do Rio de Janeiro. Resolução no 041, de 01 de julho de 2014. Disponível em:

http://www.crmvrj.org.br/wp-content/uploads/2014/07/Resolu\%C3\%A7\%C3\%A3o-CRMV-RJ-0412014.pdf. Acesso em: 23 de abril de 2020.

CFMV - Conselho Federal de Medicina Veterinária. Resolução no 1.275, de 25 de junho de 2019. Disponível em: http://www.in.gov.br/web/dou/-/resolucao-n-1.275-de-25-de-junho-de-2019203419719. Acesso em: 25 de abril de 2020.

DAVIS, M. M.; AQUILANO, N. J.; CHASE, R.B. Fundamentos da Administração da Produção. 3.ed. Porto Alegre: Bookman Editora, 2001.

FGV - Fundação Getúlio Vargas. Apoio à revisão do Plano Diretor de Desenvolvimento Urbano do Município de Niterói. Anexo IV: caderno de mapas. 2015. Disponível em: http://urbanismo.niteroi.ri.gov.br/wp-content/uploads/2015/10/diagnostico-tecnico-volume-3-

3 caderno de mapas.pdf. Acesso em: 19 de abril de 2020. 
FIGUEIREDO, K. S. A incorporação do espaço urbano pelo setor imobiliário na cidade de Niterói (RJ) e a questão da localização e das forças monopólio. Ensaios de Geografia, v. 4, p. 49-69, 2015. https://doi.org/10.22409/ensaiosgeo2015.v4i8.a21973

FONTENELLE, T. H.; CORRÊA, W. B. Uso e cobertura do solo (1976-2011) e os desafios do planejamento urbano-ambiental integrado na região oceânica de Niterói. Revista GeoNORTE, v. 3, n. 4, p. 1345-1357, 2012.

FONTENELLE, T. H.; CORRÊA, W. B. Urbanização efetiva e densidade de domicílios na região oceânica de Niterói (RJ) entre 1976 e 2010. Caminhos de Geografiaa, v. 14, n. 45, p. 172-182, 2013.

GRIECO, E. P.; PORTUGAL, L. S. Taxas de geração de viagens em condomínios residenciais em Niterói - estudo de caso. Transportes, v. 8, n. 1, p. 87-95, 2010. https://doi.org/10.14295/transportes.v18i1.386

GRISOTTI M. Doenças infecciosas emergentes e a emergência das doenças: uma revisão conceitual e novas questões. Ciência e Saúde Coletiva, v. 15, n. 1, p. 1095-1104, 2010. https://doi.org/10.1590/S1413-81232010000700017

IBGE - Instituto Brasileiro de Geografia e Estatísticas. Censo 2010. Disponível em: $<$ http://www.censo2010.ibge.gov.br/>. Acesso em: 15 de janeiro de 2020.

IBGE - Instituto Brasileiro de Geografia e Estatísticas. Cidades: Niterói. Disponível em: https:// cidades.ibge.gov.br/brasil/rj/niteroi. Acesso em: 17 de setembro de 2020.

LEFEBVRE, H. O direito à cidade. São Paulo: Centauro: 2001.

LOPES, Y. G.; ALMEIDA, A. T. Enfoque multicritério para a localização de instalações de serviço: aplicação do método SMARTER. Revista Eletrônica Sistemas \& Gestão, v. 3, n. 2, p. 114-128, 2008.

LOVISI, T. P.; SEABRA, V. S. Análise de dados censitários e temáticos na caracterização da ocupação urbana dos bairros de Niterói. Revista Continentes (UFRRJ), v. 5, n. 8, p. 6-20, 2016.

MELLO, J. A. V. B.; MELLO, A. J. R. Fundamentos de localização sob a perspectiva do consumo nas cidades. X SEGET Simpósio de Excelência em Gestão e Tecnologia. Resende - Rio de Janeiro. Outubro de 2013. 20 Disponível em: http://www.aedb.br/seget/arquivos/artigos09/540 540 artigo comercio v2.pdf. Acesso em: 23 de março de 2020.

NITERÓI. Lei no 3.385, de 21 de janeiro de 2019. Disponível em: http://pgm.niteroi.ri.gov.br/legislacao pmn/2019/Leis/Lei\%203385\%20Plano\%20Diretor.pdf. Acesso em: 22 de abril de 2020.

OEA - LA ORGANIZACIÓN DE LAS NACIONES UNIDAS PARA LA ALIMENTACIÓN Y LA AGRICULTURA; OIE - LA ORGANIZACIÓN MUNDIAL DE SANIDAD ANIMAL; OMS LA ORGANIZACIÓN MUNDIAL DE LA SALUD. Adopción de un enfoque multisectorial "Una Salud": guía tripartita para hacer frente a las enfermedades zoonóticas en los países. 2019. Disponível em: $\quad$ https://www.oie.int/es/para-los-periodistas/una-sola-salud/control-de-los-riesgossanitarios/colaboracion-nacional/. Acesso em: 8 de setembro de 2020.

OLIVEIRA, M. P.; MIZUBUTI, S. Do local ao global: jogo político, paisagem e construção de uma nova identidade para a cidade - Niterói, RJ, Brasil. In: Anais do XI Encuentro de Geógrafos de América Latina, 2007. Bogotá: Universidad Nacional de Colombia, 2007. v. 1.

PEROBELLI, F. S.; CARDOSO, V. S.; VALE, V. A.; RODRIGUES, L. C. Localização do setor de serviços e sua relação com questões espaciais no Brasil: uma análise a partir do censo demográfico de 2010. Revista Brasileira de Economia de Empresas, n. 16, v. 1, p. 53-77, 2016.

PNUD - PROGRAMA DAS NAÇÕES UNIDAS PARA O DESENVOLVIMENTO. Atlas do desenvolvimento humano. 2013. Disponível em: http://www.atlasbrasil.org.br/2013/pt/perfil/niteroi_rj. Acesso em: 20 de março de 2020.

RIBEIRO, J. C.; SANTOS, J. F. A localização dos serviços. In: Costa, J.S. (Org.). Compêndio de Economia Regional. Coimbra: APDR, 2005. p.12-21.

SANTANA, P. Acessibilidade e Utilização dos serviços de saúde: ensaio metodológico em Geografia da Saúde. Coimbra: CCRC/ARSC, 1995.

$\begin{array}{lllll}\text { Caminhos de Geografia } \quad \text { Uberlândia-MG } & \text { v. 22, n. } 79 \quad \text { Fev/2021 } & \text { p. 321-335 Página } 334\end{array}$ 
SANTANA, P. Introdução à Geografia da Saúde: território, saúde e bem-estar. Coimbra: Imprensa da Universidade de Coimbra, 2014. https://doi.org/10.14195/978-989-26-0727-6

SANTOS, M. 0 trabalho do geógrafo no Terceiro Mundo. 4 ed. São Paulo: Hucitec, 1996.

SANTOS, M. A Natureza do Espaço: técnica e tempo, razão e emoção. 4. ed. São Paulo: Edusp, 2006.

SIMOES, R.; GUIMARAES, C.; GODOY, N.; VELLOSO, T.; ARAUJO, T.; GALINARI, R. Rede urbana da oferta de serviços de saúde: uma análise espacial multivariada para Minas Gerais. In: Anais do XI Seminário sobre a Economia Mineira. Belo Horizonte: CEDEPLAR-UFMG, 2004.

ZANELLA, J. R. C. Zoonoses emergentes and reemergentes e sua importância para a saúde e a produção animal. Pesquisa agropecuária, v. 51, n. 5, p. 510-519, 2016. https://doi.org/10.1590/S0100-204X2016000500011

Recebido em: 25/04/2020

Aceito para publicação em: 06/11/2020 